\title{
CONSIDERAÇÕES SOBRE A EVAPOTRANSPIRAÇÃO ESTIMADA PELO ALGORITMO SEBAL NO SEMIÁRIDO BRASILEIRO
}

\begin{abstract}
Jório Bezerra Cabral Júnior'1 ; Rebecca Luna Lucena $^{2}$; Helder Jose Farias da Silva ${ }^{3}$; Jean Souza dos Reis ${ }^{4}$; Daniele Tôrres Rodrigues ${ }^{5}$
\end{abstract}

${ }^{1}$ Doutor em Ciências Climáticas, lotado no Instituto de Geografia, Desenvolvimento e Meio Ambiente (IGDEMA), Universidade Federal de Alagoas (UFAL), Maceió/AL, Brasil.

ORCID: https://orcid.org/0000-0002-4207-2155

Email: jorio.cabral@gmail.com

${ }^{2}$ Doutora em Geografia, lotada no Departamento de Geografia do Centro de Ensino Superior do Seridó (DGC/CERES), Universidade Federal do Rio Grande do Norte (UFRN), Caicó/RN, Brasil.

ORCID: https://orcid.org/0000-0003-4670-265X

Email: rebeccaosvaldo@yahoo.com.br

${ }^{3}$ Doutor em Ciências Climáticas, Bolsista PNPD no Programa de Pós-Graduação em Ciências Climáticas (PPGCC), Universidade Federal do Rio Grande do Norte (UFRN), Natal/RN, Brasil.

ORCID: https://orcid.org/0000-0002-0044-7270

Email: helderlagoia@gmail.com

${ }^{4}$ Mestre em Ciências Climáticas, Bolsista de Doutorado no Programa de Pós-Graduação em Ciências Climáticas (PPGCC), Universidade Federal do Rio Grande do Norte (UFRN), Natal/RN, Brasil.

ORCID: https://orcid.org/0000-0002-1674-7518

Email: jean.dosreis@hotmail.com

${ }^{5}$ Doutora em Ciências Climáticas, lotada no Departamento de Estatistica, Universidade Federal do Piauí (UFPI), Teresina/PI, Brasil.

ORCID: https://orcid.org/0000-0003-4307-2832

Email: mspdany@yahoo.com.br

\section{Resumo}

O objetivo principal neste trabalho foi avaliar a estimativa da evapotranspiração pelo algoritmo SEBAL (surface energy balance algorithm for land) no semiárido brasileiro. Para isso foi feito um levantamento bibliográfico acerca do tema, para diagnosticar se há convergência sobre a eficiência da utilização do SEBAL na aplicação e planejamento dos recursos hídricos, fazendo-se análise comparativa de diversos estudos publicados no meio científico. Os principais resultados encontrados convergiram que o SEBAL é um bom método, pois o erro oscila suavemente comparado as estimativas convencionais, além de permitir larga escala geográfica, análise com alta resolução e baixo custo. Considera-se que o SEBAL, embora tenha limitações com interferências atmosféricas, pode ser utilizado, o que se recomenda é que admita a margem de erro e que se faça análises com séries históricas para validar estatisticamente os resultados.

Palavras-chave: sensoriamento remoto; meteorologia; água.

\section{METHODOLOGICAL EVALUATION OF EVAPOTRANSPIRATION ESTIMATED BY THE SEBAL ALGORITHM IN THE BRAZILIAN SEMI-ARID}

\begin{abstract}
The main objective of this work was to evaluate the evapotranspiration estimate by the SEBAL algorithm (surface energy balance algorithm for land) in the Brazilian semiarid region. For this, a bibliographic survey was made on the subject, to diagnose if there is convergence on the efficiency of the use of SEBAL in the application and planning of water resources, making a comparative analysis of several studies published in the scientific environment. The main results found converged that SEBAL is a good method, since the error oscillates smoothly compared to conventional estimates, in addition to allowing large geographic scale, high resolution and low cost analysis. It is considered that SEBAL, although it has limitations with atmospheric interference, can be used, what is recommended is to admit the margin of error and to carry out analyzes with historical series to statistically validate the results.
\end{abstract}

Keywords: remote sensing; meteorology; water. 


\section{EVALUACIÓN METODOLÓGICA DE LA EVAPOTRANSPIRACIÓN ESTIMADA POR EL ALGORITMO SEBAL EN LA SEMIÁRIDA BRASILEÑA}

\section{Resumen}

El objetivo principal de este trabajo fue evaluar la estimación de evapotranspiración mediante el algoritmo SEBAL (algoritmo de balance de energía de superficie para tierra) en la región semiárida brasileña. Para ello, se realizó una encuesta bibliográfica sobre el tema, para diagnosticar si existe convergencia sobre la eficiencia del uso de SEBAL en la aplicación y planificación de los recursos hídricos, realizando un análisis comparativo de varios estudios publicados en el entorno científico. Los principales resultados encontrados convergieron en que SEBAL es un buen método, ya que el error oscila suavemente en comparación con las estimaciones convencionales, además de permitir una gran escala geográfica, alta resolución y análisis de bajo costo. Se considera que SEBAL, aunque tiene limitaciones con la interferencia atmosférica, puede utilizarse, lo que se recomienda es admitir el margen de error y realizar análisis con series históricas para validar estadísticamente los resultados.

Palabras-clave: teledetección; meteorología; agua.

\section{INTRODUÇÃO}

A Evapotranspiração (ET) é a perda simultânea da água por evaporação no solo e de transpiração das plantas (THORNTHWAITE, 1941; OMETTO, 1981), consome 2/3 da precipitação terrestre global (JAYAWARDENA, 1989; CHAHINE, 1992; OKI; KANAE, 2006; FISHER et al., 2017), controla o balanço de água em superfície (BHATTARAI et al., 2017a, 2017b), portanto, exerce um papel de destaque entre as variáveis mais importantes do ciclo hidrológico (HUO et al., 2013; WANG et al., 2014; XU et al., 2017).

As variações espaço-temporais da ET ocorrem em função de uma combinação de variáveis meteorológicas, cujos conhecimentos servem como indicadores hidroclimáticos essenciais para o uso eficiente dos recursos hídricos, manejo da vegetação, requisitos agrícolas e planejamento para irrigação (DROGUE et al., 2004; CHEN et al., 2007; HAN; HU, 2012; HAN et al., 2014; SHAN et al., 2015; CABRAL JÚNIOR; BEZERRA, 2018; CABRAL JÚNIOR et al., 2019). Um dos impactos sérios na relação dessas variações, por exemplo, é quando a demanda atmosférica por ET tende a ser muito maior que a oferta (precipitação), ou seja, indicador de déficit hídrico no solo.

Quanto maior for o déficit hídrico do solo numa região maior será a redução na produtividade biológica e econômica dos ecossistemas terrestres e isso representa uma séria ameaça aos processos ecológicos, biogeoquímicos e hidrológicos (MARENGO; BERNASCONI, 2015). Eleva-se também os riscos de escassez hídrica, energética e de segurança alimentar (EAKIN

et al., 2014; MEDEIROS et al., 2021). A agricultura (principalmente a de sequeiro), por exemplo, é altamente afetada.

Em regiões de clima semiárido o agravante é maior devido aos escassos recursos hídricos e a irrigação passa a ser o maior usuário de água (LIANG et al., 2010). Nessa situação, a população rural mais pobre é a mais vulnerável a este fenômeno climático (MARENGO et al., 2017; CABRAL JUNIOR; LUCENA, 2020).

Com o avanço sobre o conhecimento da perda potencial de água de uma superfície para a atmosfera (ETP) foi um dos mais significativos avanços na área de Climatologia, tendo contribuído para o planejamento em diversas áreas da ciência (Hidrologia, Agronomia, Geografia, e outros). E foi introduzido em 1944 por Thornthwaite, quando trabalhava com problemas de irrigação, no México (MATHER, 1958).

Mais tarde, na década de 1960, Monteith, com base no método de Penman, propôs um novo método que estimava diretamente a demanda energética da atmosfera por água, em condições padronizadas, denominando-o de Penman-Monteith; e Doorenbos e Pruitt (1977) introduziram o conceito de evapotranspiração de referência (ETo), em substituição ao termo ETP. Mas foi em 1990 que houve uma grande revolução sobre a proposição da conceituação e estimativa da ETo.

Neste ano, no período de 28 a 31 de maio, a Food and Agriculture Organization (FAO) promoveu, em Roma, Itália, um encontro de especialistas na área, para atender a vários objetivos, dentre eles o de analisar os conceitos e procedimentos de metodologias de cálculos da ET, com enfoque no estabelecimento de uma nova definição para a cultura de referência e o método que pudesse estimar a ET, para esta referência (Pereira et al. 1997).

Quase uma década depois Bastiaanssen et al. 1998a,b desenvolveram um dos mais destacados algoritmos do sensoriamento remoto, muito utilizado em estudos dos fluxos de calor latente e sensível à superfície, denominado pela sigla SEBAL - Surface Energy Balance Algorithm for Land. O SEBAL é um algoritmo que utiliza imagens de satélite e poucos dados de superfície para estimar os fluxos de calor latente, sensível e no

solo (SILVA, LOPES; AZEVEDO, 2005). Portanto, sugere-se que esse método possua resultados mais precisos e de baixo custo.

De acordo com Uda et al. (2013) para melhor verificação da acurácia dessa metodologia aplicada, haveria a necessidade de obtenção de medições em superfície no momento coincidente com o de aquisição da imagem de satélite, e que nesse sentido o SEBAL possui um grande potencial de uso, uma vez que permite verificar a variabilidade espacial dos componentes do balanço de radiação e energia com imagens de alta resolução espacial.

No Nordeste brasileiro, o SEBAL tem sido aplicado no semiárido por alguns pesquisadores e comparado a outros métodos convencionais. Nesse contexto, o objetivo principal neste trabalho foi avaliar, a partir de trabalhos realizados por diferentes pesquisadores, se a estimativa da ET pelo algoritmo SEBAL, no semiárido brasileiro, apresenta consenso quanto a sua acurácia e eficiência.

\section{METODOLOGIA}

Há diversas metodologias para quantificar e/ou até mesmo estimar a Evapotranspiração (ET), que são discutidas na ciência no intuito de estabelecer um método mais preciso, de custo econômico baixo e que se possa regionalizar em maiores escalas geográficas. Nesse sentido, para o alcance dos objetivos deste 
trabalho, foi realizada pesquisas bibliográficas que consistiram na análise de artigos, livros e demais produções científicas, que abordaram a temática da ET e seus métodos de análise. No decorrer dos resultados são citados autores que analisaram a ET através do algoritmo SEBAL, com destaque para aplicações em regiões semiáridas , especialmente a do Nordeste brasileiro.

\subsection{Estimativa da Evapotranspiração pelo Algoritmo} SEBAL

Na escolha de um método para a determinação da evapotranspiração, devem ser levados em consideração praticidade e precisão, pois, apesar de esses métodos teóricos e micrometeorológicos serem baseados em princípios físicos, apresentam limitações, principalmente quanto à instrumentação, o que pode restringir a utilização (BERLATO; MOLION, 1981).

A estimativa da evapotranspiração de perímetros irrigados em escala regional, com base em imagens de satélite e algoritmos que permitem a conversão de medidas instantâneas em totais diários de evapotranspiração, representa uma grande contribuição (BASTIAANSSEN et al., 1998A,B; BASTIAANSSEN, 2000;
GRANGER, 2000). Um dado indispensável à utilização do sensoriamento remoto com tal finalidade é o saldo de radiação, principal fonte de energia a ser repartida no aquecimento do solo, do ar e no processo de evapotranspiração.

A densidade de fluxo de calor no solo - $G$ é altamente dependente da condição, textura e ocupação do solo e no SEBAL o mesmo é determinado em função do NDVI - Normalized Difference Vegetation Index, temperatura da superfície, albedo e Rn. (BEZERRA; SILVA; FERREIRA, 2008).

Esse algoritmo tem sido utilizado para estimar, de forma simples e eficaz, o albedo e a temperatura da superfície com imagens Landsat 5 (BASTIAANSSEN et al., 1998A,B; DUBAYAH, 1992; GRANGER, 2000; BOEGH et al., 2002; SILVA et al., 2004) e AVHRR- NOAA (WYDICK et al., 1987; HUCEK; JACOBOWITZ, 1995; SONG; GAO, 1999; LIANG, 2002).

Observa-se o passo-a-passo inicial, na Figura 1, dos componentes do Balanço Geral de Radiação à Superfície, como procedimento fundamental para entender o processo de troca de energia na superfície e a atmosfera, e em que a Evapotranspiração é estimada como resultado final, e são indispensáveis para se estimar a ET pelo SEBAL.

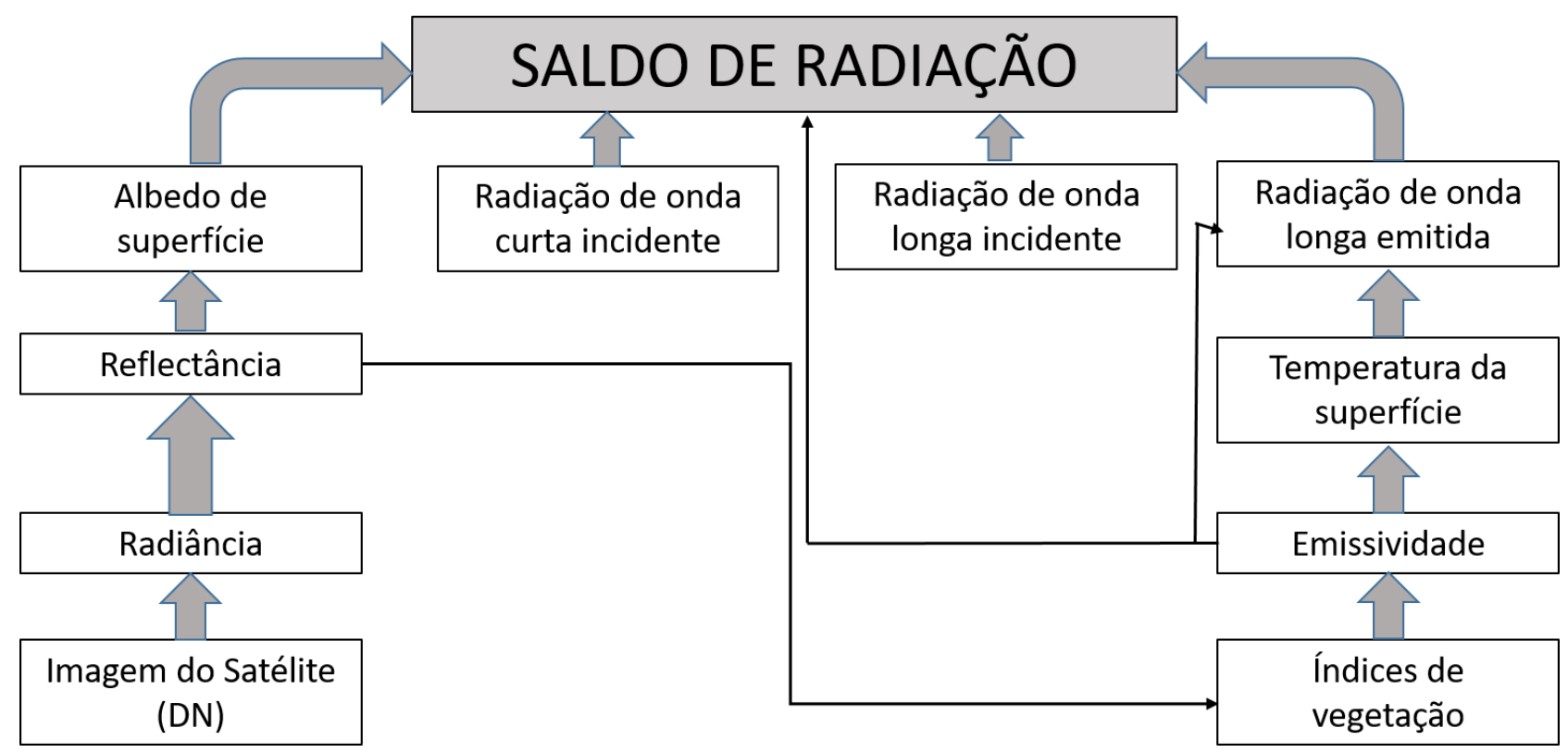

Figura 1 - Diagrama das etapas computacionais do processamento de obtenção do saldo de radiação à superfície - Rn, para estimar a Evapotranspiração. Adaptado de Silva et al. (2011).

Para a obtenção dos parâmetros acima citados é utilizada, no algoritmo SEBAL, uma rotina de equações baseadas principalmente no balanço de energia ou saldo de radiação à superfície $\left(R_{n}\right)$. Sendo:

$$
L E=R_{n}-G-H
$$

Em que: $L E$ - fluxo de calor latente, W.m-2

$R_{n}$ - saldo de radiação à superfície, W.m-2

$G$ - fluxo de calor no solo, W.m. ${ }^{-2}$, e

$H$ - fluxo de calor sensível, W.m ${ }^{-2}$.

O $R n$ é obtido através da seguinte equação: 


$$
R_{n}=Q_{g}(1-r)+\left(Q_{a}-Q_{s}\right)
$$

Sendo: $Q_{g}$ - radiação de onda curta incidente, $\mathrm{W} . \mathrm{m}^{-2}$; $r$ - albedo da superfície, adimensional;

$Q_{a}$ - radiação de onda longa incidente, W. $\mathrm{m}^{-2}$; $Q_{s}$ - radiação de onda longa emitida, W.m ${ }^{-2}$;

Destaca-se que para determinação do fluxo de calor no solo $(G)$ é calculada a razão $G / R_{n}$. Allen et al. (2002) recomendam que em superfícies líquidas essa razão seja de $0,5\left(G / R_{n}=0,5\right)$, para os demais pixels é utilizada a seguinte equação:

$$
\left.\frac{G}{R_{n}}=[T \underset{s}{(0,0038}+0,0074 r)\left(1-0,98 N D V I^{4}\right)\right]
$$

Em que: $G$ - fluxo de calor no solo, $\mathrm{W} \mathrm{m}^{-2}$; $R_{n}$ - saldo de radiação à superfície, $\mathrm{W} \mathrm{m}^{-2}$; $T_{s}$ - temperatura da superfície, ${ }^{\circ} \mathrm{C}$; $r$ - albedo da superfície, adimensional; NDVI - índice de vegetação (adimensional);

O cômputo do fluxo de calor sensível $(H)$ é realizado através da equação:

$$
H=\frac{\rho C_{p} d T}{r_{a h}}
$$

Sendo: $H$ - fluxo de calor sensível;

$\rho$ - massa específica do ar úmido, $1,15 \mathrm{~kg} \mathrm{~m}^{-3}$;

$C_{p}$ - calor específico do ar, $1005 \mathrm{~J} \mathrm{~kg}^{-1} \mathrm{~K}^{-1}$;

$d T$ - diferença de temperatura entre a superfície e a região imediatamente superior, $\mathrm{K}$, e

$r_{a h}$ - resistência aerodinâmica ao transporte de calor, $\mathrm{s} \mathrm{m}^{-1}$.

As etapas envolvidas para determinação da ET deixam claro que os principais produtos do SEBAL estão o saldo radiação $\left(R_{n}\right)$, fluxo de calor do solo $(G)$ e fluxo de calor latente $(L E)$, sendo os mesmos calculados para cada pixel da imagem. Ressalta-se que a principal vantagem do SEBAL é a necessidade de dados mínimos no solo (FOLHES et al., 2009).

Para Moreira et al. (2010), além de estimar a ET e os termos do balanço de energia na superfície, o algoritmo SEBAL permite detectar a existência de áreas degradadas ou em processo de degradação. Áreas com NDVI muito baixo, albedo elevado e baixa ET indicam áreas degradadas, de solo exposto ou pouco vegetadas e secas.

\section{RESULTADOS E DISCUSSÃO}

Alguns trabalhos têm sido realizados para verificar a acurácia do SEBAL, na região semiárida do Nordeste do Brasil, e tem-se encontrado resultados significativos.

Ayenew (2003) aplicou o SEBAL na estimativa da evaporação de lagos e da evapotranspiração diária de superfícies vegetadas, utilizando dados do sensor Advanced Very High Resolution Radiometer (AVHRR), na região semiárida da Etiópia. Este autor citou que as diferenças espaciais dos resultados do SEBAL na superfície ficaram de acordo com as estimativas de métodos de evapotranspiração convencional.

$\mathrm{O}$ algoritmo Sebal também tem sido muito utilizado por autores brasileiros (SILVA; BEZERRA, 2006; FOLHES, 2009; MENDONÇA, 2007; BEZERRA et al., 2008; NICÁCIO, 2008), para estimar valores de evapotranspiração e encontraram resultados consistentes, quando comparados com os medidos em campo, com variações entre 1 e $10 \%$ no resultado final.

Bezerra et al. (2008) analisaram o método no semiárido brasileiro, estado do Ceará, e encontraram que na reserva florestal da Chapada do Araripe, a ET apresentou praticamente o mesmo comportamento, bastante uniforme. A ET foi estimada pelo algoritmo SEBAL aplicado a imagens TM - Landsat 5 e seus resultados foram comparados com resultados obtidos pela técnica de razão de Bowen. As diferenças apresentadas, tanto para valores horários como para diários, foram inferiores a 10\%, demonstrando, dessa forma, a potencialidade da técnica.

Silva et al (2012) analisando um perímetro irrigado no semiárido paraibano, concluíram que as estimativas da ET com uso do algoritmo SEBAL, apresentam boa concordância com os valores da evapotranspiração obtidos com o método da FAO.

Santos, Fontana e Alves (2010) apontaram o modelo SEBAL com uso do sensor ASTER é eficiente para estimar os fluxos de energia em superfície e a ET diária em regiões de cultivo de arroz.

Mesmo com esses trabalhos já publicados na região do

semiárido, ainda não foi possível fazer análises de séries históricas, que permitam consolidar os dados aplicando testes estatísticos. Entretanto, a técnica do SEBAL pode ser utilizada em regiões onde haja a ausência de medições em estação meteorológica padronizada. Ainda é insuficiente as comparações realizadas para substituir plenamente métodos convencionais com

o SEBAL na Estimativa da ET, entretanto ressalta-se que o referido algoritmo tem apresentado desempenhos satisfatórios em estudos pontuais.

Corroborando com Cavalho et al. (2011, p. 462), "embora a evapotranspiração, sob o aspecto conceitual, possa ser relativamente simples, quando se parte para os aspectos práticos de sua medição e estimativa, ainda nota-se a dificuldade e complexidade em apresentar uma solução que seja plenamente satisfatória e, portanto, continuam surgindo constantes estudos, visando a dar uma resposta mais consistente ao problema".

A utilização de sensoriamento remoto representa uma alternativa interessante para a obtenção do padrão espacial de variáveis hidrológicas. A vantagem de utilizar o sensoriamento remoto para obter a evapotranspiração, é que sua estimativa pode ser obtida exclusivamente por meio da equação de balanço de energia, não sendo necessário se conhecer dados acerca da cobertura vegetal e do teor de água no solo (WARREN, 2013). 
Mais recentemente Cavalcante et al. (2016) concluíram, para uma intensa área de produtividade agrícola no semiárido nordestino (Juazeiro-BA e Petrolina-PE), que o algoritmo SEBAL apresentou considerável acurácia. Esses autores recomendaram sua aplicação como uma forma economicamente viável e por abranger maior área de informações, se comparadas a métodos tradicionais.

\section{CONSIDERAÇÕES FINAIS}

Através da pesquisa realizada, foi possível constatar que o Algoritmo SEBAL apresenta boa estimativa, e, portanto é uma técnica que pode ser utilizada na estimativa da ET, embora, apresente limitações, dentre elas a estimativa em dias nebulosos, o que dificulta o monitoramento cronológico por este método.

Vale destacar que o conhecimento acerca da ET é fundamental para o monitoramento hídrico na agricultura e em reservatórios hídricos, principalmente em regiões semiáridas, a exemplo do semiárido brasileiro, uma vez que essa variável é a componente principal de perda de água da superfície e depende da demanda energética da atmosfera e da água disponível superficialmente.

Recomendam-se, para melhor efeito de validação, análises com maior número de observações de estimativa de ET pelo SEBAL, comparados aos métodos convencionais observados em superfície, para que possam ser feitos testes estatísticos e determinar a significância de sua utilização.

\section{REFERÊNCIAS}

AB'SÁBER, A. N. Problemática da Desertificação e da Savanização no Brasil Intertropical. São Paulo, IGEOG/USP, 1977.

ALLEN, R.G.; TREZZA, R.; TASUMI, M. Surface energy balance algorithms for land - Advance training and users manual, version 1.0. Idaho: Kimberly, 2002.

BHATTARAI, N., QUACKENBUSH, L.J., IM, J., SHAW, S.B. A new optimized algorithm for automating endmember pixel selection in the SEBAL and METRIC models. Remote Sens. Environ., v. 196, 178-192, 2017a.

BHATTARAI, N., WAGLE, P., GOWDA, P.H., KAKANI, V.G. Utility of remote sensingbased surface energy balance models to track water stress in rain-fed switchgrass under dry and wet conditions. ISPRS J. Photogramm. Remote. Sens., v.133, p.128-141, 2017b.

BERLATO, M. A.; MOLION, L.C.B. Evaporação e evapotranspiração. Porto Alegre: IPAGRO/Secretaria de Agricultura, 1981. 95 p. (Boletim Técnico, 7).

BEZERRA, B. G.; SILVA, B. B. da; FERREIRA, N. J.; Estimativa da Evapotranspiração real diária utilizando-se imagens digitais TM - LANDSAT 5. Revista Brasileira de Meteorologia, v.23, n.3, p. 305-317, 2008.
BISHT G. et al. Estimation of the net radiation using MODIS (Moderate Resolution Imaging Spectroradiometer) data for clear sky days. Remote Sensing of Environment, v. 97, n. 1, p. 52-67, 2005.

BRANCO, Samuel Murgel. Água: origem, uso e preservação. São Paulo: Moderna, 2003.

CABRAL JÚNIOR, J. B.; BEZERRA, B. G. Análises da evapotranspiração de referência e do índice de aridez para o Nordeste do Brasil. Revista de Geociências do Nordeste, v. 4, n. 1, p. 71-89, 2018.

CABRAL JUNIOR, J. B.; LUCENA, R. L. Analysis of precipitation using Mann-Kendall and Kruskal-Wallis NonParametric Tests. Mercator (Fortaleza), v.19, 2020.

CABRAL JÚNIOR, J.B., SILVA, C.M.S.E., ALMEIDA, H.A.; BEZERRA, B. G.; SPYRIDES, M. H. C. Detecting linear trend of reference evapotranspiration in irrigated farming areas in Brazil's semiarid region. Theor Appl Climatol, 138, 215-225 2019.

CAMARGO, A. P. de; CAMARGO. M. B. P. de. Uma revisão analítica da evapotranspiração potencial. Bragantia, Campinas, 59(2), 125-137, 2000.

CARVAlHO, L. G. de, RIOS, G. F. A, MIRANDA, W. L, NETO, P. C. Evapotranspiração de referência: uma abordagem atual de diferentes métodos de estimativa. Pesq. Agropec. Trop., Goiânia, v. 41, n. 3, p. 456-465, jul./set. 2011.

CHAHINE, M.T. The hydrological cycle and its influence on climate. Nature, v.359, n.6394, p.373-380, 1992.

CHEN, H.; GUO, S.L.; XU, C.Y.; SINGH, V.P. Historical temporal trends of hydro-climatic variables and runoff response to climate variability and their relevancein water resource management in the Hanjiang basin. Journal of Hydrology, v.344, n.3-4, p.171-184, 2007.

CONTI, J. B. A questão climática do Nordeste brasileiro e os processos de desertificação. São Paulo, Revista Brasileira de Climatologia, Vol. 1, Nº 1, 2005.

DOORENBOS, J.; PRUITT, W. O. Crop water requirements. Rome: FAO, 1977. (Irrigation and drainage paper, 24).

DROGUE, G.; PFISTER, L.; LEVIANDIER, T.; IDRISSI, A.E.; IFFLY, J.F.; MATGEN, P.; HUMBERT, J.; HOFFMANN, L. Simulating the spatio-temporal variability of streamflowresponse to climate change scenarios in a mesoscale basin. Journal of Hydrology, v.293, n.1-4, p.255269, 2004.

EAKIN, H.C.; LEMOS, M.C,; NELSON, D.R. Differentiating capacities as a means to sustainable climate change 
adaptation. Global Environmental Change, v. 27, p. 1-8, 2014.

FOLHES, M.T. Modelagem da evapotranspiração para a gestão hídrica de perímetros irrigados com base em sensores remotos. Tese (Doutorado) Instituto Nacional de Pesquisas Espaciais, São José dos Campos, 2007.189p.

FOLHES, M.T. RENNÓ; C.D.; SOARES; J.V. Remote sensing for irrigation water management in the semi-arid Northeast of Brazil. Agricultural Water Management, v.96, p. 13981408, 2009.

FEITOSA, J. R. P. Uso de Técnica de Sensoriamento Remoto e Imagens Landsat -TM e NOAA-AVHRR na Estimativa do Balanço de Radiação à Superfície. 164f. Tese (Doutorado em Recursos Naturais). Programa Institucional de Pósgraduação em Recursos Naturais. Universidade Federal de Campina Grande. Campina Grande, 2005.

FISHER, J. B.; MELTON, F.; MIDDLETON, E.; et al. The future of evapotranspiration: Global requirements for ecosystemfunctioning, carbon and climate feedbacks, agriculturalmanagement, and water resources. Water Resources Research, v. 10.1002/20, p. 2618-2626, 2017.

FOLHES, M.T.; RENNO, C.D.; SOARES , J.V. Remote sensing for irrigation water management in the semi-arid Northeast of Brazil. Agricultural Water Management, v. 96, p. 13981408, 2009.

GIACOMONI, M. H.; MENDES, C. A. B.; Estimativa da Evapotranspiração Regional por meio de Técnicas de Sensoriamento Remoto Integradas a Modelo de Balanço de Energia. Revista Brasileira de Recursos Hídricos. V. 13, n.4, p. 33-42, 2008.

GONDIM, R. S.; FUCK JÚNIOR, S. C. F.; EVANGELISTA, S. R. M. Castro, M. A. H. de,; Teixeira, A. S. Impacto das Mudanças Climáticas na Evapotranspiração em Nível de Bacia Hidrográfica Utilizando um Sistema de Informações Geográficas. Revista Brasileira de Recursos Hidricos v. 16 n.2, p. 5-12, 2011.

HAN, S.J.; HU, H.P. Spatial variations and temporal changes in potential evaporation in the Tarim Basin, northwest China (1960-2006): influenced by irrigation? Hydrological Processes, v.26, n.20, p.3041-3051, 2012.

HAN, S.J.; TANG, Q.H.; XU, D.; WANG, S.L. Irrigationinduced changes in potential evaporation: more attention is needed. Hydrological Processes, v.28, n.4, p.2717-2720, 2014.

HERDIES, D.L.; PEZZI, L. P.; ARAVÉQUIA, J. A. Centro de Previsão do Tempo e Estudos Climáticos-CPTEC. Instituto de Pesquisas Espaciais - INPE.
JAYAWARDENA, A.W. Calibration of some empirical equations for evaporation and evapotranspiration in Hong Kong. Agricultural and Forest Meteorology, v.47, n.1, p.7581,1989 .

LIANG, L.; LI, L.; LIU, Q. Temporal variation of reference evapotranspiration during 1961-2005 in the Taoer River basin of Northeast China. Agricultural and Forest Meteorology, v. 150, n. 2, p. 298-306, 2010.

MAO, J.; FU, W.; SHI, X.; RICCIUTO, D. M.; FISHER, J. B.; DICKINSON, R. E.; WEI, Y.; SHEM, W.; PIAO, S.; WANG, K. et al., Disentangling climatic and anthropogenic controls on global terrestrial evapotranspiration trends, Environmental Research Letters, v.10, n.9, p.094008, 2015.

MARENGO, J. A. Biodiversidade: caracterização do clima atual e definição das alterações climáticas para o território brasileiro ao longo do século XXI. Brasília: MMA: 2006.

MARENGO, J. A.; BERNASCONI, M. Regional differences in aridity/drought conditions over Northeast Brazil: present state and future projections. Climatic Change, v. 129, n. 12, p. 103-115, 2015.

MARENGO, J. A.; TORRES, R. R.; ALVES, L. M. Drought in Northeast Brazil - past, present, and future, Theoretical and Applied Climatology, v. 129, n. 3-4, p. 1189-1200, 2017.

MATHER, J.R. Preface. Centerton, N.J.,. (Publications in Climatology, v. XI, n.3). 1958, p. 247-248.

MEDEIROS, F. J.; OLIVEIRA, C. P.; GOMES, R. S.; SILVA, M. L.; CABRAL JÚNIOR, J. B. Hydrometeorological conditions in the semiarid and east coast regions of Northeast Brazil in the 2012-2017 period. Anais da Academia Brasileira de Ciências, v. 93, n. 1, p. 1-15, 2021.

MENDONÇA, J.C. Estimação da evapotranspiração regional utilizando imagens digitais orbitais na região Norte Fluminense, RJ. Tese (Doutorado) Universidade Estadual do Norte Fluminense Darcy Ribe iro, Campo dos Goytacazes. 2007. 167p.

MOREIRA, L. C. J.; DURAND, B. J.; TEIXEIRA, A. S.; ANDRADE, E. M.; Variabilidade local e regional da Evapotranspiração estimada pelo algoritmo SEBAL. Eng. Agríc., Jaboticabal, v.30, n.6, p.1148-1159, 2010.

MOURA, A. R. C.; MONTENEGRO, S. M. G. L.; ANTONINO, A. C. D.; AZEVEDO, J. R.G. de.; SILVA, B. B. da; OLIVEIRA, L. M. M. de.; Evapotranspiração de referência baseada em métodos empíricos em bacia experimental no estado de Pernambuco - Brasil. Revista Brasileira de Meteorologia, v.28, n.2, p. 181 - 191, 2013.

NICÁCIO, R.M. Evapotranspiração real e umidade do solo usando dados de sensores orbitais e a metodologia SEBAL na bacia do rio São Francisco. Tese 
(Doutorado). Universidade Federal do Rio de Janeiro, Rio de Janeiro, 2008. 337p.

OKI, T.; KANAE, S. Global hydrological cycles and world water resources, Science, v. 313, n. 5790, p. 1068-1072, 2006.

OMETTO, J. C. Bioclimatologia vegetal. São Paulo, SP, Ed. Agronômica Ceres, 440p. 1981.

PEREIRA, A. R.; VILlA NOVA, N. A.; SEDIYAMA. G.C. Evapo(transpi)ração. Piracicaba: Fealq, 1997.

REBOITA, M. S. et al. Causas da semi-aridez do sertão nordestino. Revista brasileira de Climatologia. Ano $12-$ Vol. 19 - JUL/DEZ. 254-277 p. 2016.

REBOUÇAS, A. C. (Org). Águas doces no Brasil: capital ecológico, uso e conservação. Brasil: Escrituras Editora, 2002.

SANTOS, T. V. dos; FONTANA, D. C.; ALVES, R. C. M. Avaliação de fluxos de calor e evapotranspiração pelo modelo SEBAL com uso de dados do sensor ASTER. Pesq. agropec. bras., Brasília, v.45, n.5, p.488-496, 2010.

SHAN, N.; SHI, Z.; YANG, X.; GAO, J.; CAI, D. Spatiotemporal trends of reference evapotranspiration and its driving factors in the Beijing-Tianjin Sand Source Control Project Region, China. Agricultural and Forest Meteorology, v. 200, 2015.

SILVA, B.B. da; BEZERRA, M.V.C. Determinação dos fluxos de calor sensível e latente na superfície utilizando imagens TM - Landsat 5. Revista Brasileira de Agrometeorologia, v.14, p.174- 186, 2006.

SILVA, B. B.; BRAGA, A. C.; BRAGA, C. C. Balanço de radiação no perímetro irrigado São Gonçalo-PB mediante imagens orbitais. Revista Caatinga, v.24, n.3, 145-152, 2011.
SILVA, B. B. da; BRAGA, A. C.; BRAGA, C. C.; OLIVEIRA, L. M. M. de; GALVÍNCIO, J. D.; MONTENEGRO, S. M. G. L.; Evapotranspiração e estimativa da água consumida em perímetro irrigado do Semiárido brasileiro por sensoriamento remoto. Pesq. agropec. bras., Brasília, v.47, n.9, p.1218-1226, 2012.

SUDENE - Superintendência do Desenvolvimento do Nordeste. Delimitação do semiárido. Disponível em: http://www.sudene.gov.br/delimitacao-do-semiarido. Acesso em: 6 julho. 2010.

THORNTHWAITE, C.W.; HOLZMAN, B. Evaporation and transpiration. In: Climate and Man: Yearbook of Agriculture. Washington D.C : U.S. Department of Agriculture, p.545-550, 1941.

WANG, L.; CAO, L.; DENG, X.; et al. Changes in aridity index and reference evapotranspiration over the central and eastern Tibetan Plateau in China during 1960-2012. Quaternary International, v. 349, p. 280-286, 2014.

WARREN, M. S. Desagregação espacial de estimativas de evapotranspiração real obtidas a partir do sensor Modis. Revista Brasileira de Meteorologia, v.28, n.2, 153 - 162, 2013.

XU, Y.; XU, Y.; WANG, Y.; et al. Spatial and temporal trends of reference crop evapotranspiration and its influential variables in Yangtze River Delta, eastern China. Theoretical and Applied Climatology, v. 130, n. 3-4, p. 945-958, 2017.

Recebido em: 10/10/2019

Aceito para publicação em: 23/05/2021 